\title{
Sugammadex versus Neostigmine in Reversing Neuromuscular Block Induced by Rocuronium during Adenotonsillectomy in Pediatric Patients
}

\author{
Ashraf M Moustafa, Osama A EISharkawy, Ashraf M AlKassas, Ahmed M Abo Ali \\ Anesthesia and Intensive Care Department, Faculty of Medicine, Menoufiya University, Egypt.
}

Background and aim of the work: Neuromuscular blocking agents (NMB) are still indispensable for surgical procedures requiring general anesthesia. Higher doses may be necessary to reach the same NMB level in children, as in adults. The neuromuscular junction in infants is not sufficiently mature. All these factors lead to an increased risk of postoperative apnea in pediatric patients. At this point an NMB reversing agent with a reduced postoperative residual curariation (PORC) risk is of great importance. Cholinesterase inhibitors are traditionally used for reversal of neuromuscular blockade. Among these agents neostigmine is the most potent and selective one.

Today, sugammadex is an alternative to the decurarization procedure, which was traditionally executed with cholinesterase inhibitors. PORC and the muscarinic side effects are not anticipated when using sugammadex, which has been developed so as to be specific for rocuronium and vecuronium. Sugammadex is proved to be a safe and superior agent in NMB reversal compared to neostigmine in adults. However especially in pediatric cases, it is seen that the studies about its reliability and side effects are limited. The aim of this study was to compare sugammadex and neostigmine as regards efficacy, safety and side effects in pediatric patients undergoing adenotonsillectomy.

Patients and Methods: Fourty pediatric patients undergoing elective adenotonsillictomy operation with standrized propofol-fentanyl-rocuronium-isoflurane anesthetic technique were devided randomly into two equal groups $(n=20)$ either receive sugammadex $2 \mathrm{mg} / \mathrm{kg}$ or neostigmine $0.03 \mathrm{mg} / \mathrm{kg}$ and atropine $0.01 \mathrm{mg} / \mathrm{kg}$. At the end of the operation when $\mathrm{T} 2$ appeared by train of four (TOF) watch "SX model acceleromyograph" monitor, tested drug was given. When TOF ratio $>\mathbf{9 0 \%}$ was reached, patients were extubated. Time to reach TOFR $>90 \%$ after reverse, hemodynamic alteration and side effects were recorded and compared.

Results: Patients in sugammadex group attained a TOF ratio $90 \%$ was statistically significantly shorter time ( $88 \pm 45.4$ seconds) than those in neostigmine group $(415.8 \pm 227.5$ seconds) $(p=0.0002)$, heart rate was statistically significantly higher at 2, 5 and 10 minutes in neostigmine group compared with baseline $(p<0.01)$. Comparison of adverse effects yielded no difference "vomiting in $3 / 20(15 \%)$ patients with sugammadex and $4 / 20(20 \%)$ patients with neostigmine, and $1 / 20(5 \%)$ patient developed

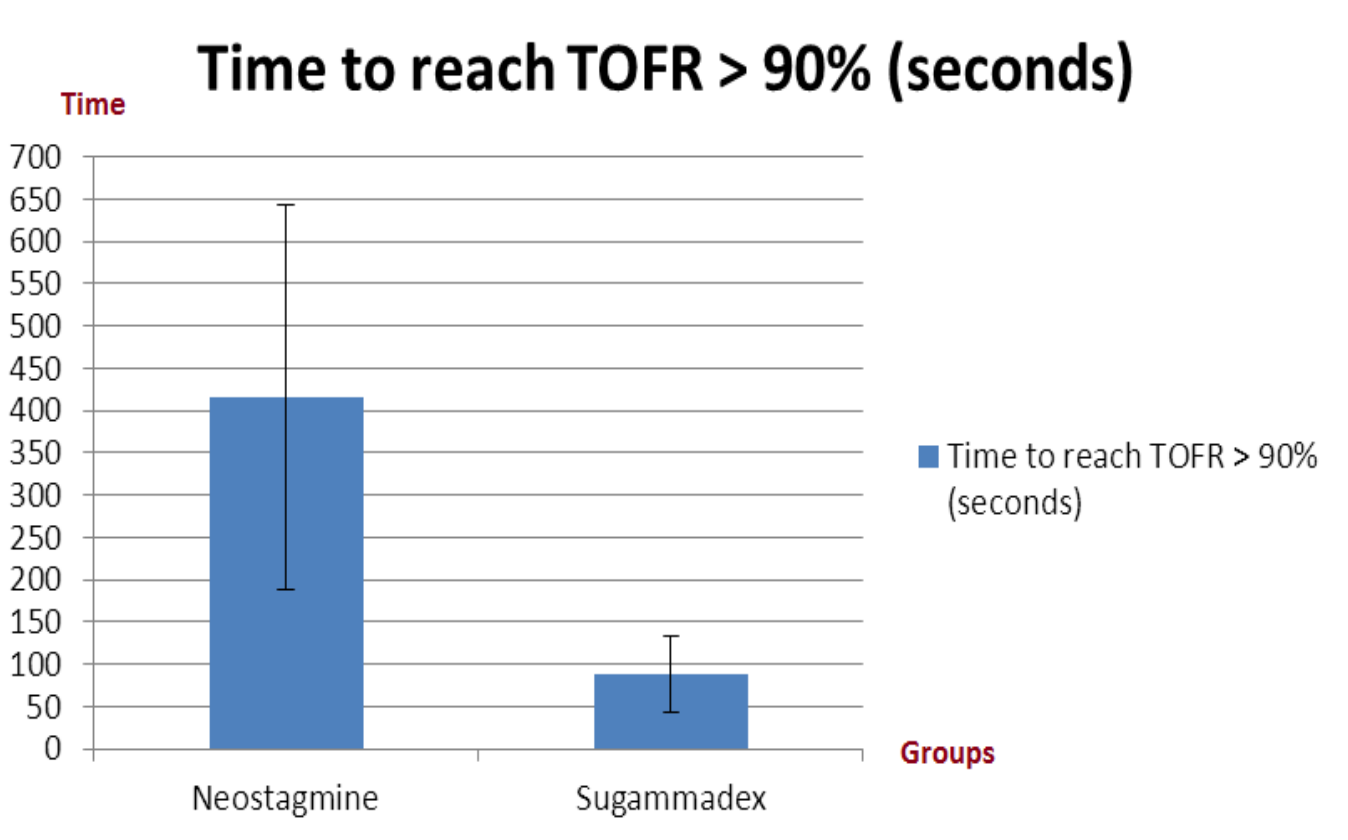
desaturation in both groups".

Conclusion: Sugammadex is a new selective relaxant binding agent that rapidly and effectively reverses rocuronium-induced neuromuscular blockade in pediatric patients, and was well tolerated overall in the children when administered at a dose $2 \mathrm{mg} / \mathrm{kg}$ at the reappearance of T2 of TOF with comparable adverse effects.

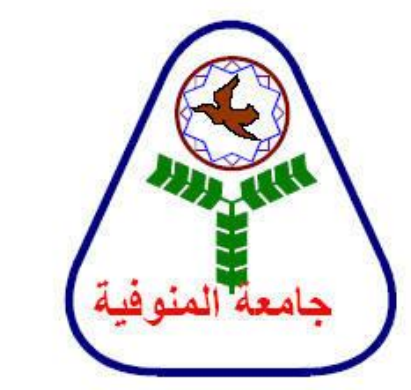

